\title{
IP Controller Design for Uncertain Two-Mass Torsional System Using Time-Frequency Analysis
}

\author{
Jing Cui, ${ }^{1}$ Jiayu Ye, ${ }^{1}$ and Zhongyi Chu $\mathbb{D}^{2}$ \\ ${ }^{1}$ College of Mechanical Engineering and Applied Electronics Technology, Beijing University of Technology, Beijing 100022, China \\ ${ }^{2}$ School of Instrument Science and Opto-Electronics, Beihang University, Beijing 100191, China \\ Correspondence should be addressed to Zhongyi Chu; chuzystar@gmail.com
}

Received 20 July 2017; Revised 28 November 2017; Accepted 26 February 2018; Published 29 March 2018

Academic Editor: Naveed Ahmad

Copyright (c) 2018 Jing Cui et al. This is an open access article distributed under the Creative Commons Attribution License, which permits unrestricted use, distribution, and reproduction in any medium, provided the original work is properly cited.

\begin{abstract}
With the development of industrial production, drive systems are demanded for larger inertias of motors and load machines, whereas shafts should be lightweight. In this situation, it will excite mechanical vibrations in load side, which is harmful for industrial production when the motor works. Because of the complexity of the flexible shaft, it is often difficult to calculate stiffness coefficient of the flexible shaft. Furthermore, only the velocity of driving side could be measured, whereas the driving torque, the load torque, and the velocity of load side are immeasurable. Therefore, it is inconvenient to design the controller for the uncertain system. In this paper, a low-order IP controller is designed for an uncertain two-mass torsional system based on polynomial method and time-frequency analysis (TFA). IP controller parameters are calculated by inertias of driving side and load side as well as the resonant frequency based on polynomial method. Therein, the resonant frequency is identified using the time-frequency analysis (TFA) of the velocity step response of the driving side under the open-loop system state, which can not only avoid harmful persistent startstop excitation signal of the traditional method, but also obtain high recognition accuracy under the condition of weak vibration signal submerged in noise. The effectiveness of the designed IP controller is verified by groups of experiments. Experimental results show that good performance for vibration suppression is obtained for uncertain two-mass torsional system in a medium-low shaft stiffness condition.
\end{abstract}

\section{Introduction}

Drive systems have a pivotal position in the field of modern industry including robots, rolling mills, textile, or paper machines [1]. Most of these systems can be reduced to a model whose motor and load machine are connecting through a shaft. With the increasing levels of industrial production, these systems are demanded for larger inertias of motors and load machines, whereas shafts should be lightweight. In this case, the model is turned into a two-mass torsional system, as the shafts could no longer be regarded as rigid. Excessive shaft twists will be generated easily when the motor has just been started and exciting mechanical vibrations not only are affecting the performance of drive systems greatly, but also are able to lead to instability of the entire drive system [2].

On the other hand, for these industrial drive systems, usually the velocity of driving side can be measured, whereas the driving torque, the load torque, and the velocity of load side are immeasurable [3]. In this case, torsional vibrations of the load side must be suppressed by only using the velocity feedback of the driving side, such as using PI speed controller and additional feedback [4], $H \infty$ feedback controller and IP-position controller [5], neural network speed controller [6], and controllers based on polynomial method [7]. Herein, low-order IP controllers occupied an important position in engineering application due to the characteristics of simple structure, convenient parameters calculation, and easy application. However, the system models are generally considered to be known when controllers are designed and flexible shaft stiffness and resonant frequency of the system are given directly in the parameter list, or the resonance frequency is obtained by drawing the Bode plot based on certain openloop system model [4-6].

According to the actual engineering applications, like rolling machines, machine tools, injection molding machines, and other mechanical systems using flexible 
connection, the resonant frequency of the system is difficult to measure due to the unknown stiffness coefficient of the flexible shaft that makes the system uncertain. Therefore, it is inconvenient to design the controller with the unknown resonant frequency of the system, especially only with the measured driving side velocity.

To solve this problem, many studies of frequency identification had been implemented: the Fast Fourier Transform (FFT) is one of the most widely used and well-established methods for frequency domain analysis. Nonetheless, owing to the characteristic of the velocity step response of drive side in which tiny vibration is submerged in noise, using the FFT cannot identify the resonance frequency definitely. Meanwhile, frequency response of the system can be obtained by using Welch-Method in current and speed signal with Pseudo-Random Binary Signals (PRBS) excitation [8, 9], the measured frequency response functions (FRFs) of driveside and load-side velocities with Random Binary Signal (RBS) excitation [10]. Nevertheless, regardless of whether the excitation signal is PRBS or RBS, persistent start-stop excitation will do more harm to the system than using step signal.

In this paper, a low-order IP controller is designed based on polynomial method for uncertain two-mass torsional system. Parameters of IP controller are calculated by inertias of driving side and load side as well as the resonant frequency. Therein, owing to the uncertain system, the resonant frequency of the system as a necessary parameter for calculating IP controller parameters is recognized by using timefrequency analysis (TFA) and the conducted signal is only the velocity step response of the drive side. The effectiveness of designed IP controller is verified by groups of experiments. Experiment results show that the vibrations of the load side are well suppressed.

This paper is outlined as follows. The experimental set-up and modeling of the two-mass system are introduced in Section 2. In Section 3, the design method of IP controller for the uncertain system is briefly implemented, while it emphasizes identifying the resonant frequency of the system especially only with the measured driving side velocity. Groups of experiments are conducted in Section 4. Experiments verify that, with this approach, vibrations of the load side are suppressed very well. Finally, conclusions and future work are given in Section 5.

\section{Experimental Set-Up and Modeling of the Two-Mass System}

2.1. Experimental Set-Up. The experimental platform is shown in Figure 1. The test platform is controlled by the control programs which are written in AS 3.0.90 (B\&R Automation Studio) on a personal computer (PC). A voltage regulator module (VRM) is used to actuate the direct current (DC) brush motor. A mass which is rigidly connected with the motor (after a planetary gear reducer) can be seen as the drive side. The drive side and load side of the system are coupled by a spring as the flexible shaft. There are two same-type encoders attached to the drive side and the load side, respectively. The resolution ratio of each encoder is
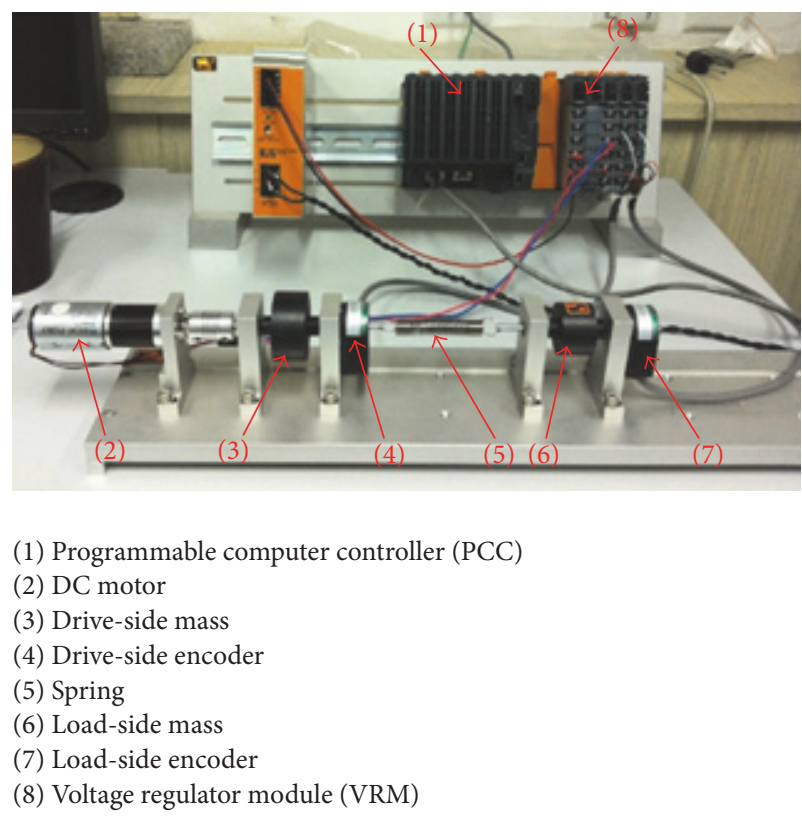

FIGURE 1: The experimental platform.

4000 pulses/rev. The drive-side encoder is used to carry out the feedback control and to gain the velocity of the drive side for identifying the resonance frequency. The performance of IP controller is reflected by the velocity of load side, which is collected by the load-side encoder. All the experiments are implemented under the sampling time of $10 \mathrm{~ms}$. The structure of the whole experimental system is shown in Figure 2.

2.2. Modeling of the Two-Mass System. The model of the twomass system can be abstracted as in Figure 3. The damping has been omitted. The following formulas can be listed according to the dynamic theory:

$$
\begin{aligned}
T_{s} & =K_{s} * \int\left(\omega_{m}-\omega_{l}\right) \\
T_{m}+T_{s} & =d \omega_{m} * J_{m} \\
T_{l}-T_{s} & =d \omega_{l} * J_{l} .
\end{aligned}
$$

$J_{m}, J_{l}$ are inertias of the drive side (including the drive motor) and the load side, respectively

$T_{m}, T_{l}$ are torques of the drive side and the load side, respectively

$\omega_{m}, \omega_{l}$ are velocities of the drive side and the load side, respectively

$T_{s}$ is torque generated by the shaft during rotation

$K_{s}$ is spring coefficient.

According to (1), the block diagram of the system can be drawn as in Figure 4. 


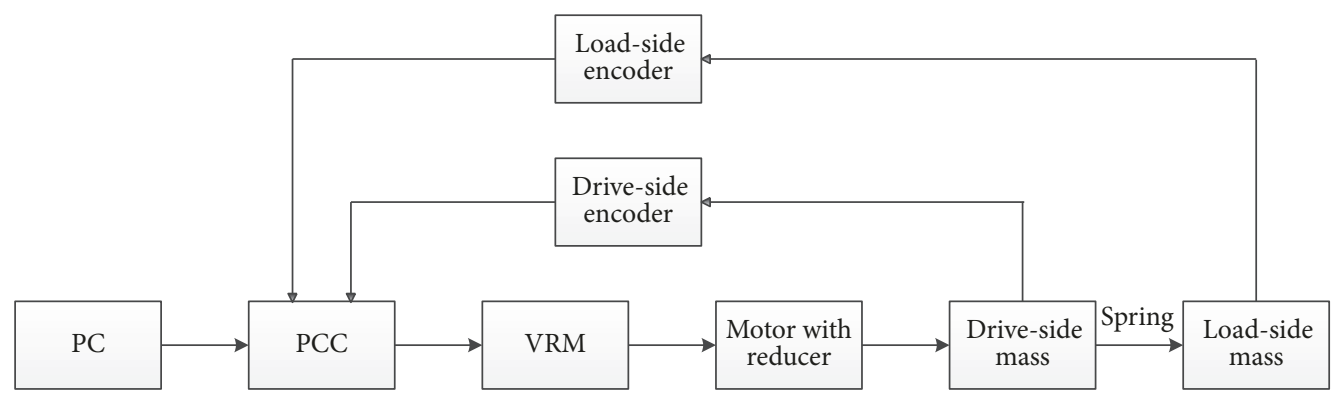

FIGURE 2: The structure of the experimental system.

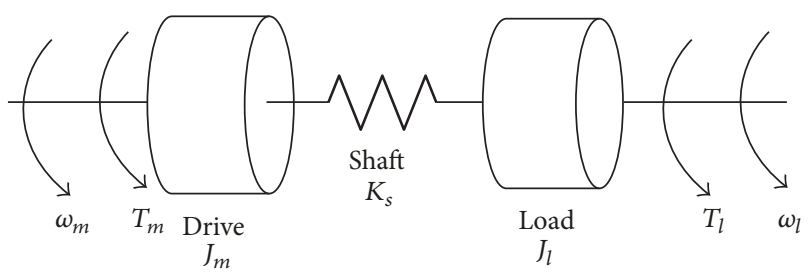

FIgURE 3: Model of the two-mass system.

From the block diagram, the transfer function between $T_{m}$ and $\omega_{m}$ can be written as

$$
P(s)=\frac{s^{2}+\omega_{\text {ares }}^{2}}{J_{m} s\left(s^{2}+\omega_{\text {res }}^{2}\right)},
$$

where $\omega_{\text {res }}$ and $\omega_{\text {ares }}$ are the resonance frequency and antiresonance frequency, respectively.

$$
\begin{aligned}
\omega_{\text {res }} & =\sqrt{\frac{K_{s}\left(J_{m}+J_{l}\right)}{J_{m} J_{l}}} \\
\omega_{\text {ares }} & =\sqrt{\frac{K_{s}}{J_{l}}} \\
\omega_{\text {ares }} & =\sqrt{q} * \omega_{\text {res }} \\
q & =\frac{J_{m}}{J_{m}+J_{l}} .
\end{aligned}
$$

$q$ is defined as the inertia ratio as shown in (6).

For a generalized discussion, (2) can be standardized by replacing the complex variable $s$ with $s^{*}=s / \omega_{\text {ares }}$.

$$
P\left(s^{*}\right)=\frac{q\left(s^{* 2}+1\right)}{J_{m} \omega_{\text {ares }}\left(q s^{* 3}+s^{*}\right)} .
$$

For the sake of simplicity, the standardized two-mass system transfer function can be taken as

$$
P_{n}\left(s^{*}\right)=\frac{s^{* 2}+1}{q s^{* 3}+s^{*}} .
$$

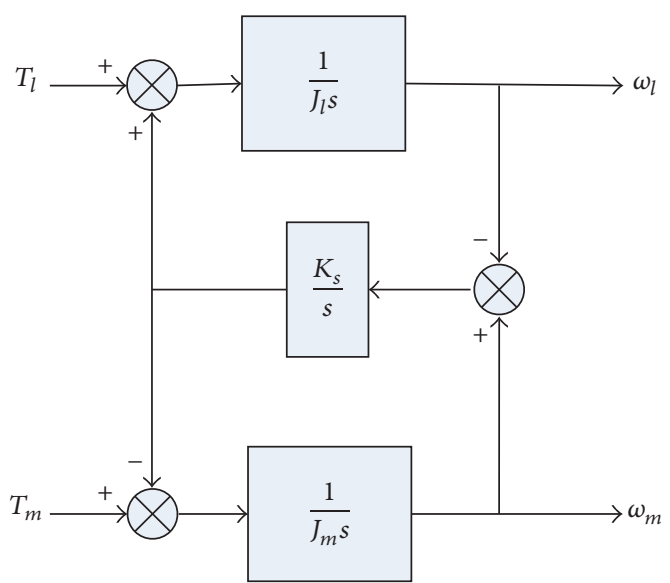

FIGURE 4: Block diagram of the two-mass system.

\section{IP Controller Design}

3.1. IP Controller Design. The block diagram of the closedloop system with IP feedback controller is shown in Figure 5. The closed-loop system transfer function can be derived from the block diagram.

$$
G_{n}\left(s^{*}\right)=\frac{K_{i}^{*}\left(s^{* 2}+1\right)}{q s^{* 4}+K_{p}^{*} s^{* 3}+\left(1+K_{i}^{*}\right) s^{* 3}+K_{p}^{*} s^{*}+K_{i}^{*}} .
$$

Comparing (7) and (8), IP controller parameters $K_{p}, K_{i}$ can be derived as

$$
\begin{gathered}
K_{p}=\frac{K_{p}^{*} J_{m} \omega_{\text {ares }}}{q} \\
K_{i}=\frac{K_{i}^{*} J_{m} \omega_{\text {ares }}^{2}}{q},
\end{gathered}
$$

where $K_{p}^{*}, K_{i}^{*}$ are the standardized controller parameters. By substituting (5) into (10), IP controller parameters $K_{p}, K_{i}$ can be rewritten as

$$
\begin{gathered}
K_{p}=\frac{K_{p}^{*} J_{m} \omega_{\text {res }}}{\sqrt{q}} \\
K_{i}=K_{i}^{*} J_{m} \omega_{\text {res }}^{2} .
\end{gathered}
$$




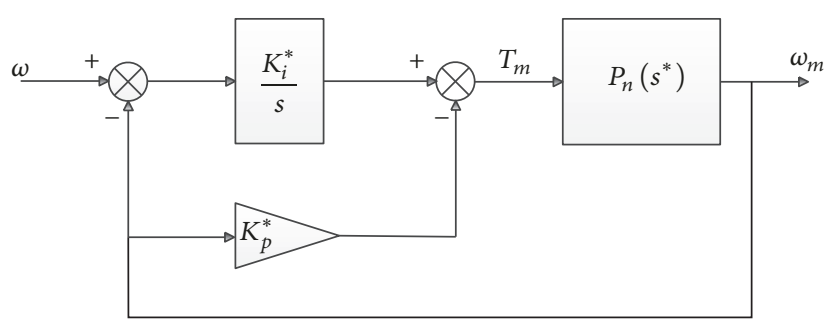

FIGURE 5: Block diagram of the closed-loop system.

According to [11, 12], IP controller is applied when inertia ratio $q<1 / 3$ and $K_{p}^{*}, K_{i}^{*}$ can be selected as follows in order to have a good performance with sufficient damping provided:

$$
\begin{aligned}
K_{p}^{*} & =\frac{4}{5 \sqrt{2}}, \\
K_{i}^{*} & =\frac{1}{4} .
\end{aligned}
$$

From (11), the inertias of drive side (including the drive motor) and load side as well as the resonance frequency are needed to calculate IP controller parameters. The inertias can be obtained by conventional calculating or 3D modeling software.

3.2. Identification of the Resonance Frequency. As mentioned in Section 1, for a two-mass torsional system, usually the velocity of the drive side can be measured. So, vibration suppression of the load side is only able to use the velocity feedback of the driving side. The velocity step responses of the drive side and the load side are given in Figures 6(a) and 6(b). Using the FFT to identify them is attempted and their results are given in Figures 6(c) and 6(d), respectively. According to the results, it is obvious that only the FFT can identify the resonance frequency from the velocity step responses of the load side (shown in Figure 6(d)). While for the drive-side velocity, it just does not have any effect (shown in Figure 6(c)). In this case, for the system in which only the drive-side velocity can be measured, the FFT does not apply to resonance frequency analysis. So, a more efficient analytical method is needed.

Because of the tiny vibration of the velocity step response of the drive side (shown in Figure 6(a)), time-frequency analysis (TFA) is used for obtaining the resonance frequency of the system by revealing the energy size of drive-side velocity in each time period. One of the most popular methods of TFA is the short-time Fourier transform (STFT) [13]. When the STFT is used, it is considered that a certain width of window sliding in the nonstationary signal is assumed to be piecewise stationary. The signal intercepted by the window is processed by the traditional Fourier transform [14]. The discrete form of STFT is able to be obtained by using the time-frequency grid point $(m \Delta t, n \Delta f)$ of equal interval sampling the signal $z(k \Delta t)$.
The signal $z(k \Delta t)$ is represented by the velocity response of the drive side, as shown in Figure 6(a):

$$
\begin{aligned}
& \operatorname{STFT}_{z}(m \Delta t, n \Delta f) \\
& \quad=\sum_{k=-\infty}^{\infty} z(k \Delta t) \eta[(m-k) \Delta t] e^{-j 2 \pi(n \Delta f) k \Delta t} \Delta t .
\end{aligned}
$$

$$
\begin{aligned}
& m \Delta t \text { is time point } \\
& n \Delta f \text { is frequency point } \\
& \Delta t \text { is sampling interval of the time variable } \\
& \Delta f \text { is sampling interval of the frequency variable } \\
& \eta(\Delta t) \text { is time window function. }
\end{aligned}
$$

It is important to choose a suitable window function for the STFT to play the best performance [15]. The narrower the main-lobe of the window, the higher the frequency resolution that can be got and the faster the attenuation of the side-lobe of the window, the less the energy leakage. Considering the signal obtained from the velocity of drive side, whose resonance point is concerned but not energy size, the Hanning-window is selected because of its characteristic of less energy leakage and better frequency resolution.

Velocity step response of the drive side as the test signal is processed by using Hanning-window in MATLAB. According to the experimental data, for example, as in Figure 6(a), the sampling frequency is set as $100 \mathrm{~Hz}$ (equivalent to $\Delta t=$ $10 \mathrm{~ms}$ in (13)) and sampling as $30 \mathrm{~s}$. In this case, tiny vibration is shown in the first 500 data points out of 3000 data points $(k=0,1,2, \ldots, 2999)$. The window width $m$ is set as 512 considering the length of the vibration signal and an optimal frequency resolution. On this basis, the overlapping width between each two windows is set as 500 in order to have a better time aggregation [12]. So, the time-frequency spectrum can be obtained as shown in Figure 7. There are two obvious yellow lines in Figure 7. The long yellow line (around $7 \mathrm{~Hz}$ ) stands for the system backlash which is formed by the connecting way of spring and load. The short one (around $1.9 \mathrm{~Hz}$ ) represents the resonant frequency which is marked in Figure 7. Comparing Figure 7 with the FFT result of the loadside velocity (shown in Figure $6(\mathrm{~d})$ ), it is testified that the STFT can effectively extract the resonance frequency from the velocity step responses of the drive side.

\section{Experiment Results}

To verify the validity of the method, experiments are carried out on the experimental platform. For the sake of simplicity, only the drive side inertia, the load side inertia, and the spring coefficient are adjustable. There are three kinds of weight of masses. Their inertias as well as other components are shown in Table 1 . The equivalent inertias of drive side $J_{m}$ and load side $J_{l}$ can be calculated as follows:

$$
\begin{aligned}
J_{m} & =J_{m 1}+J_{m 2}+J_{m 3}+J_{\text {mass }} \\
J_{l} & =J_{m 3}+J_{\text {mass }} .
\end{aligned}
$$




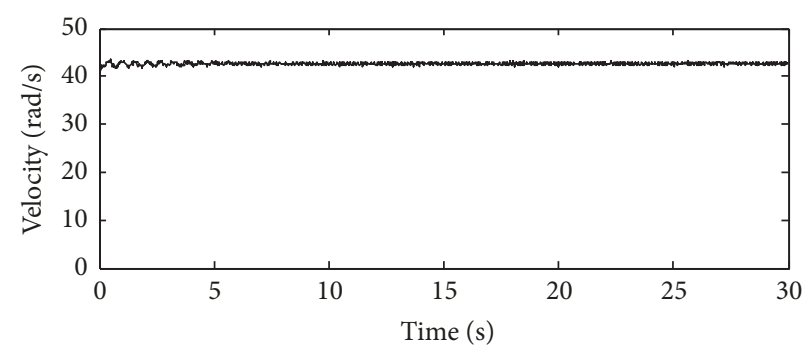

(a)

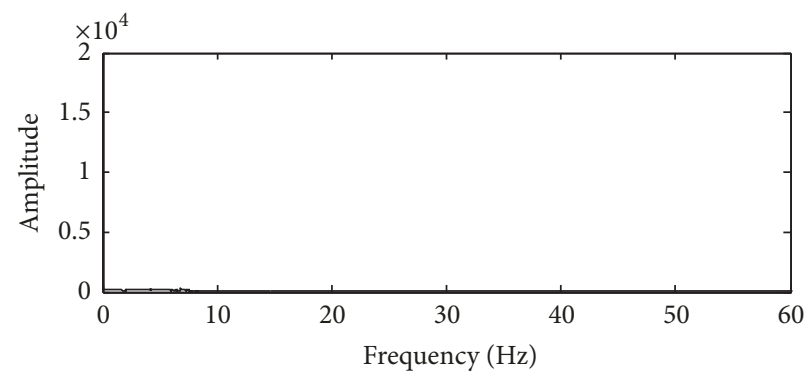

(c)

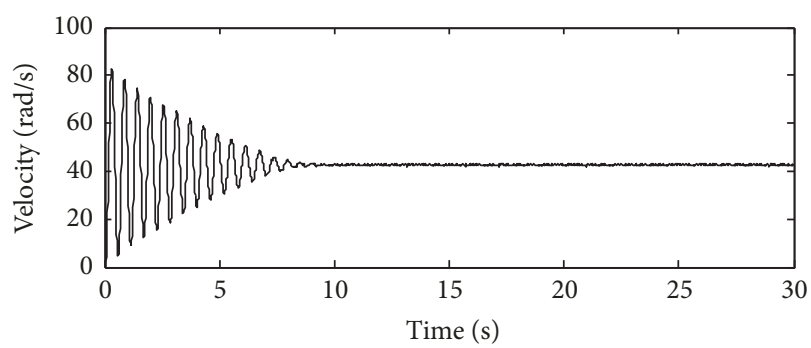

(b)

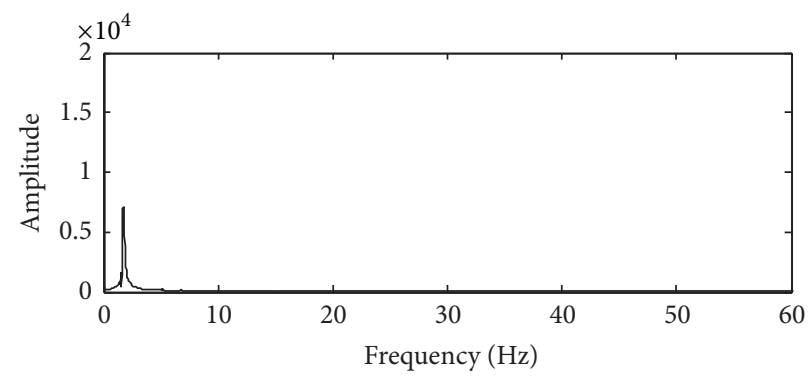

(d)

FIGURE 6: The velocity step responses of the drive side and the load side as well as their results of the FFT: (a) velocity step response of drive side, (b) velocity step response of load side, (c) FFT result of velocity step response of drive side, and (d) FFT result of velocity step response of load side.

TABLE 1: Inertias of DC motor, coupler, stiff shaft, and three masses.

\begin{tabular}{lcc}
\hline Parameter & Inertia $\left(\times 10^{-6} \mathrm{~kg} \cdot \mathrm{m}^{2}\right)$ & Description \\
\hline$J_{m 1}$ & 1.361 & Inertia of the DC motor with a reducer \\
$J_{m 2}$ & 0.562 & Inertia of the coupler \\
$J_{m 3}$ & 0.019 & Inertia of the stiff shaft \\
$J_{\text {mass }}$ & 5.303 & \\
$J_{\text {mass } 1}$ & 16.281 & Inertias of masses. $J_{\text {mass }}$ stands for any one of the three inertias \\
$J_{\text {mass } 2}$ & 44.196 & \\
$J_{\text {mass } 3}$ & & \\
\hline
\end{tabular}

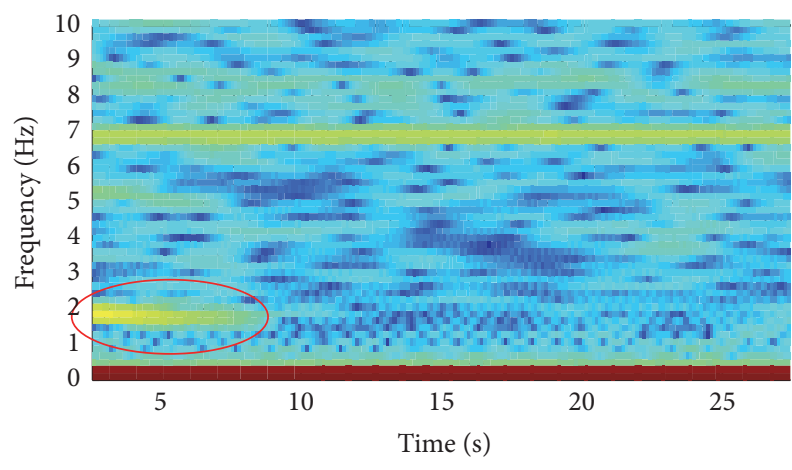

FIGURE 7: Time-frequency spectrum of the drive-side velocity using the STFT.

There are four springs of stainless steel with different sizes used in experiments to verify the applicability of this method. The sizes of springs are given in Table 2. The stiffness of springs are arrayed from small to large. The first experiment is to determine the inertia ratio $q=0.285$, by changing the
TABLE 2: Various sizes of the springs.

\begin{tabular}{lc}
\hline Number & Wire diameter $*$ outside diameter $(\mathrm{mm})$ \\
\hline$(1)$ & $0.8 * 10$ \\
$(2)$ & $1 * 10$ \\
$(3)$ & $1 * 8$ \\
$(4)$ & $1.2 * 10$ \\
\hline
\end{tabular}

different types of springs to verify the feasibility of the method mentioned in Section 3.

The time-frequency spectra of the velocity of drive side with different springs are given in Figure 8. Referring to Figure 8 , resonant frequencies are marked by red arrows with different springs and IP controller parameters $K_{p} ; K_{i}$ can be calculated by (11). Calculation results of IP controller parameters $K_{p}, K_{i}$ are given in the caption of Figure 9. The experimental velocity responses of both drive side and load side are shown in Figure 9. In the process of experiments, the stiffness of springs always remains unknown. Only the velocity response of the drive side is used when extracting 


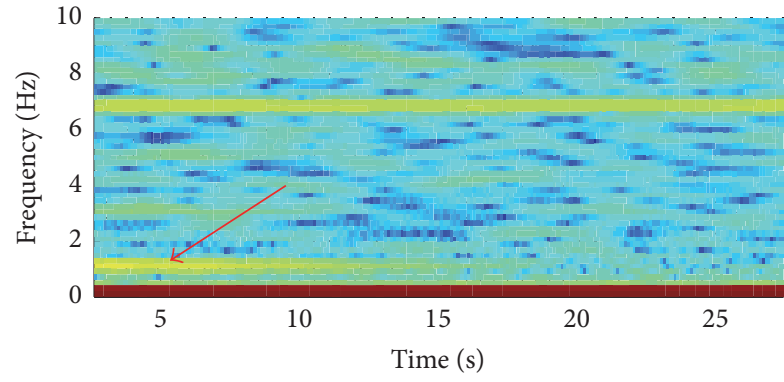

(a)

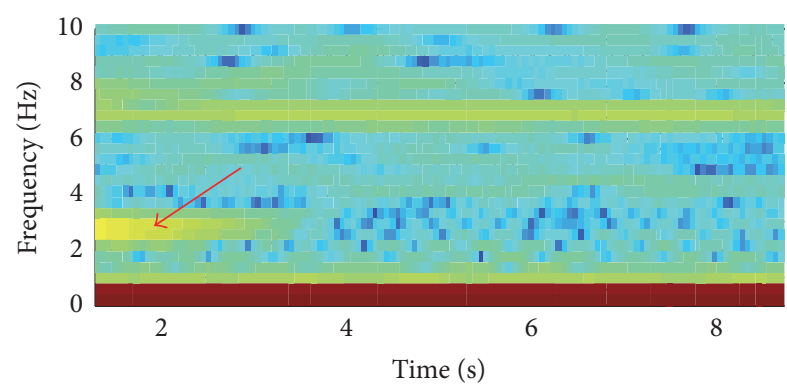

(c)

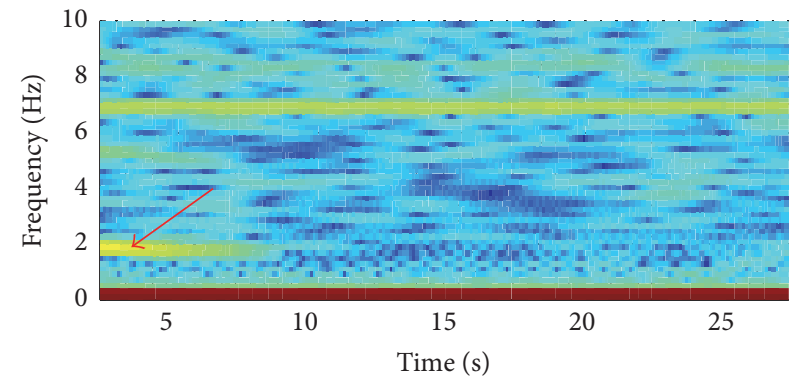

(b)

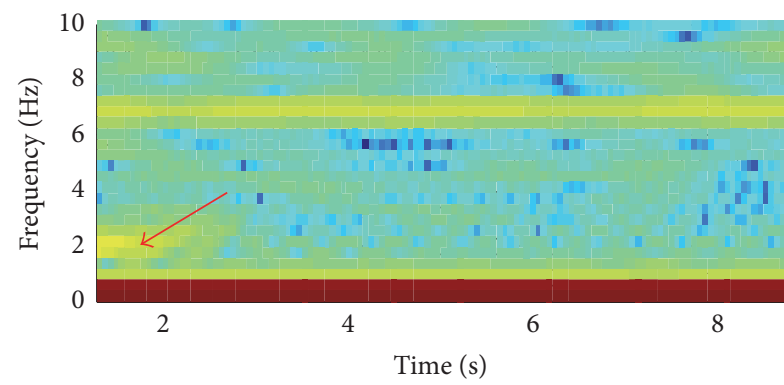

(d)

FIGURE 8: The time-frequency spectra of the velocity of drive side with different springs $(q=0.285$ ): (a) spring 1, (b) spring 2, (c) spring 3 , and (d) spring 4 .

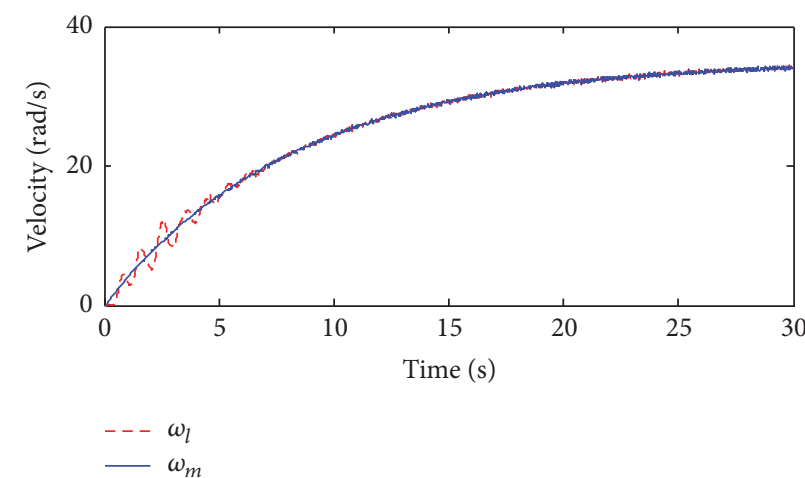

(a)

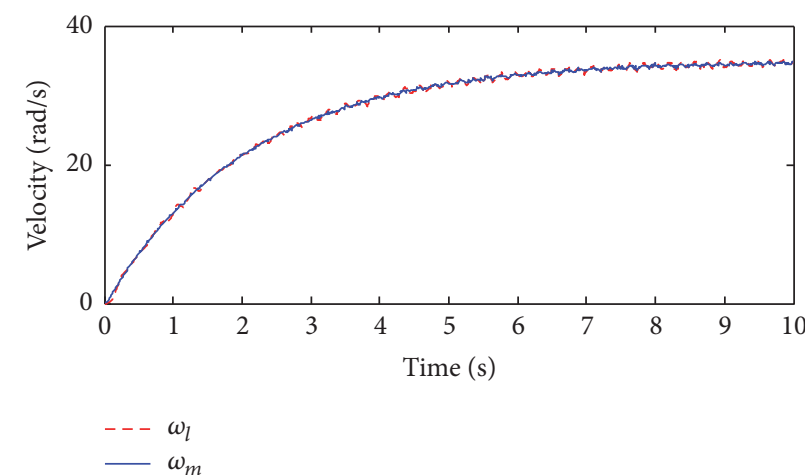

(c)

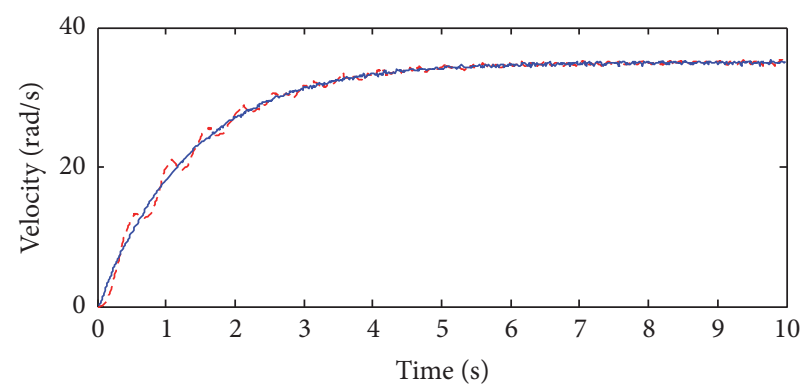

$---\omega_{l}$

$-\omega_{m}$

(b)

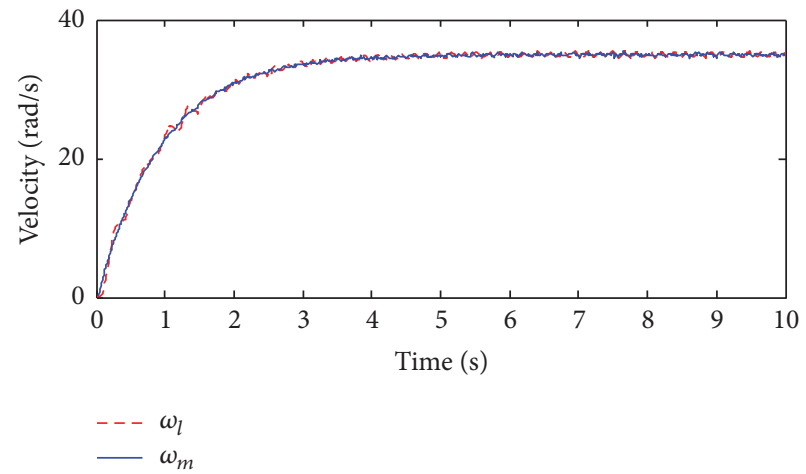

(d)

FIGURE 9: The experimental velocity responses of load side with different springs ( $q=0.285)$ : (a) spring $1, K_{p}=0.0175, K_{i}=1.5771 ;(b)$ spring $2, K_{p}=0.0276, K_{i}=3.9537$; (c) spring $3, K_{p}=0.032, K_{i}=5.3$; (d) spring $4, K_{p}=0.0393, K_{i}=7.984$. 


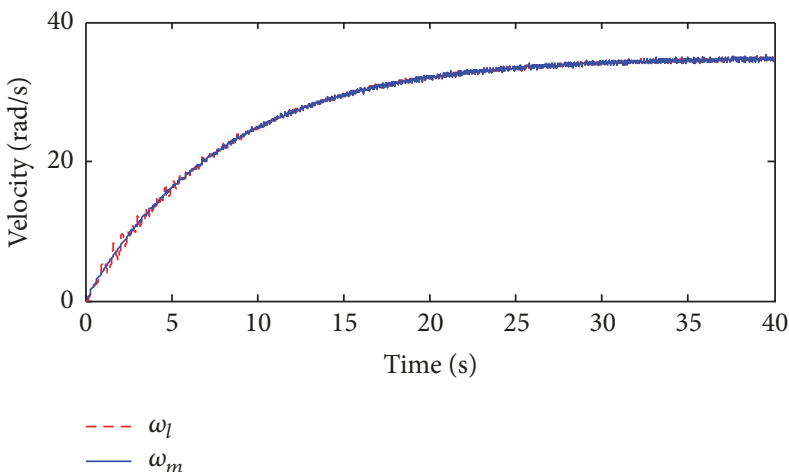

(a)

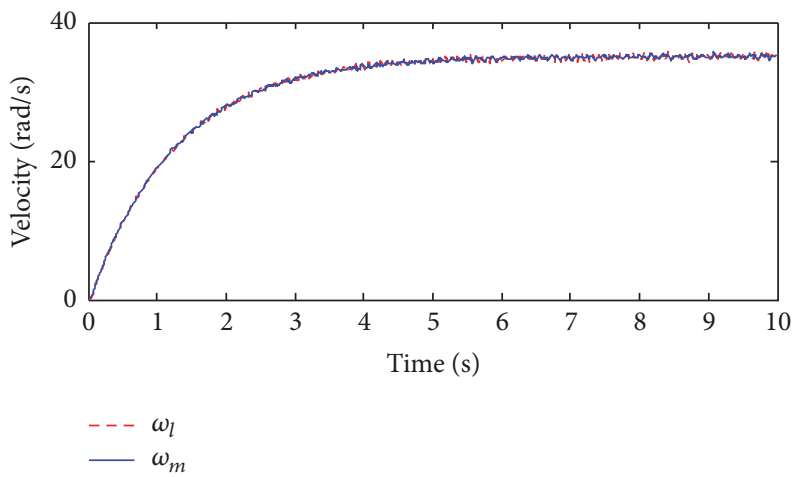

(c)

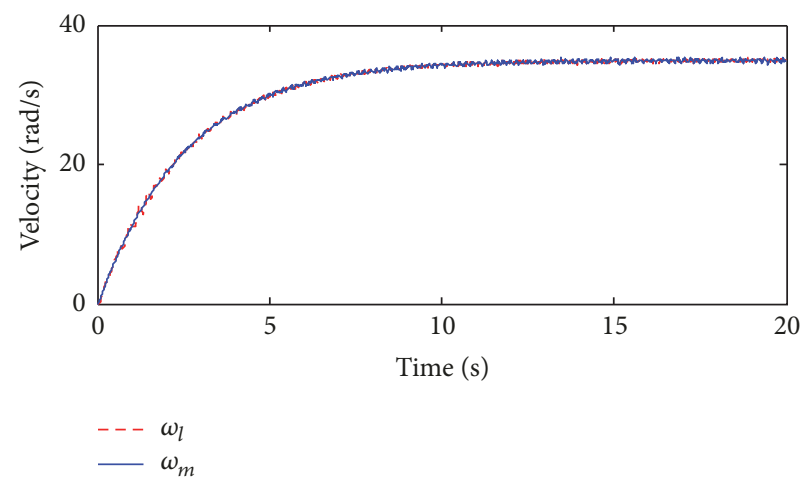

(b)

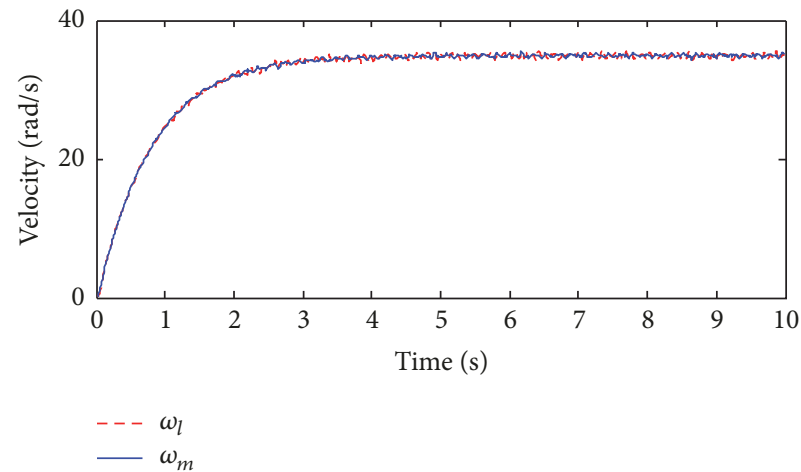

(d)

FIGURE 10: The experimental velocity responses of load side with different springs $(q=0.290)$ : (a) spring $1, K_{p}=0.0093, K_{i}=1.1943$; (b) spring $2, K_{p}=0.0163, K_{i}=3.7193$; (c) spring $3, K_{p}=0.0276, K_{i}=4.7772$; (d) spring $4, K_{p}=0.0397, K_{i}=9.922$.

TABLE 3: The combination of masses.

\begin{tabular}{lcc}
\hline Drive side & Load side & Inertia ratio $q$ \\
\hline Mass 2 & Mass 3 & 0.285 \\
Mass 1 & Mass 2 & 0.290 \\
Mass 1 & Mass 3 & 0.131 \\
\hline
\end{tabular}

the resonant frequency by STFT. Experimental results show nonovershoot in Figure 9. The velocity response of the load side which is sampled by a load-side encoder is almost overlapping together with the velocity response of the drive side. Nevertheless, there are still tiny vibrations when the motor is started due to the low stiffness of the springs as shown in Figures 9(a) and 9(b).

Other experiments are also conducted by changing inertia ratio $q$ and repeating the above steps. The inertia ratio $q$ can be altered by changing the mass on the drive and load sides. In order to satisfy the condition that inertia ratio $q<$ $1 / 3$, three kinds of combinations can be permitted and inertia ratio $q$ is calculated, respectively, which are given in Table 3 . The results are shown in Figures 10, 11, and 12. Although tiny vibrations exist when the motor just starts in the $5 \mathrm{~s}$ (shown in Figures 10(a), 10(b), 11(a), and 11(b)), the IP controller designed in the case of system uncertainty still shows a good performance with controller parameters designed by using the STFT. Nonovershoot is still performed and the velocity response of the load side follows up the velocity response of the drive side very well. Even then, one experiment result (shown in Figure 12) is not as expected. Because of the high shaft stiffness, the speed of vibration attenuation of the load side response is very fast (shown in Figure 12(a)). The STFT is not able to analyze the resonance frequency of the system because the vibration signal is covered by window width (shown in Figure 12(b)).

\section{Conclusion and Future Work}

In this paper, a low-order IP controller of the two-mass system with model uncertainty has been designed for vibration suppression. The controller is designed based on polynomial method. Parameters of IP controller are calculated by inertias of drive side and load side as well as the resonant frequency. In case the shaft stiffness coefficient is unknown, the resonant frequency of the system is identified by using STFT in velocity step response of the drive side under the open-loop system. The effectiveness of this method is verified through three groups of experiments by changing the inertia ratio $q$. Referring to experimental results, the designed IP controller shows good performance for vibration suppression. However, when the shaft stiffness is high, the speed of vibration attenuation of the load side response is very fast (shown in Figure 12(a)). The STFT is not able to analyze the resonance frequency of the system because the vibration signal is covered by window 


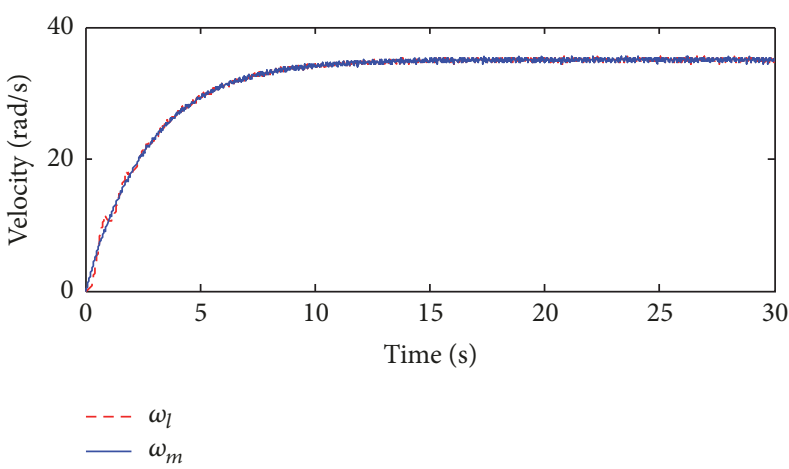

(a)

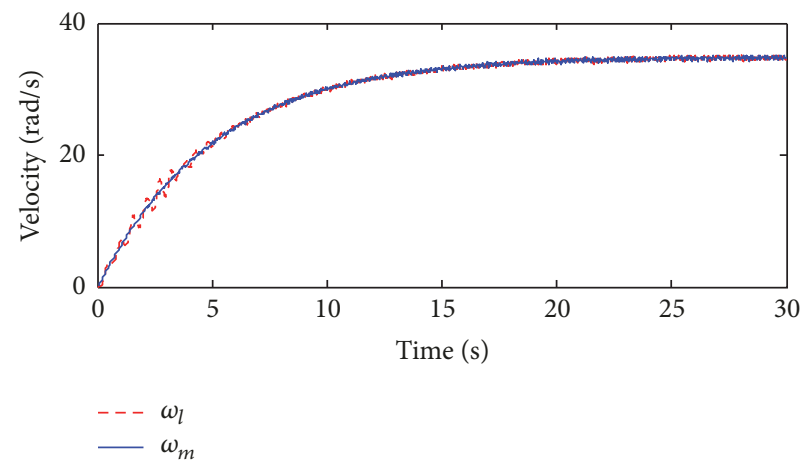

(b)

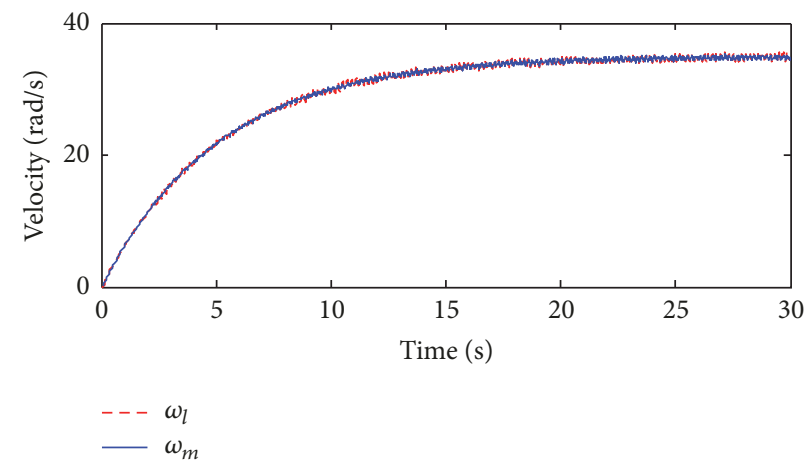

(c)

FIGURE 11: The experimental velocity responses of load side with different springs $(q=0.131)$ : (a) spring $1, K_{p}=0.0089, K_{i}=0.5$; (b) spring $2, K_{p}=0.0138, K_{i}=1.1943$; (c) spring $3, K_{p}=0.0162, K_{i}=1.653$.

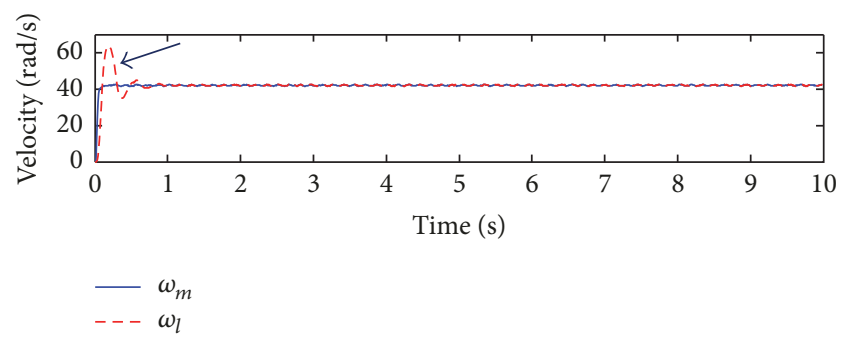

(a)

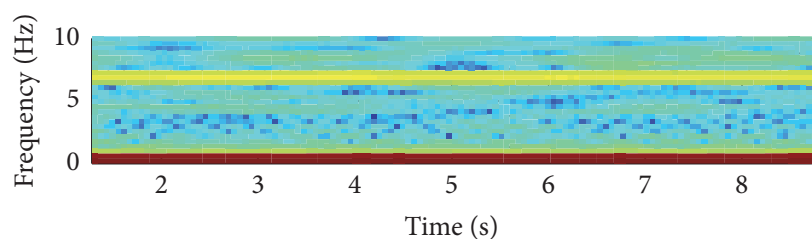

(b)

FIGURE 12: (a) Velocity response of the drive side and the load side when $q=0.131$ and spring 4 are chosen. (b) The time-frequency spectra of the velocity of the drive side.

width (shown in Figure 12(b)), while shortening the window width will decrease the frequency resolution. Therefore, the controller parameters cannot be calculated in this case.

According to the phenomenon found in this paper, in order to enhance the performance of the two-mass torsional system further, future work will focus on the following aspects. (1) First one is determining the scope of the application mentioned above quantitatively. The reason why the use of STFT has a limitation is that the window width may cover the vibration signal in settling time. In this case, encoders with higher resolution can be used to obtain more data of vibration signal. Then, the frequency resolution of STFT can be improved with wider window and parameters of a two-mass torsional system with high-stiffness shaft can be identified indirectly. (2) Other TFA methods or frequency extracting methods will be considered. For example, wavelet transform (WT) is a powerful method in signal processing. The window width of WT is variable depending on the signal frequency. Therefore, its frequency-related window can improve the frequency extraction ability in two-mass torsional system with different shaft stiffness.

\section{Conflicts of Interest}

The authors declare that there are no conflicts of interest regarding the publication of this manuscript. 


\section{Acknowledgments}

The authors acknowledge the financial support from the Natural Science Foundation of China (61773028), the Natural Science Foundation of Beijing (4172008), and the National Science and Technology Major Project of China (2013ZX04008021).

\section{References}

[1] W. Li and Y. Hori, "Vibration suppression using single neuronbased PI fuzzy controller and fractional-order disturbance observer," IEEE Transactions on Industrial Electronics, vol. 54, no. 1, pp. 117-126, 2007.

[2] X. Xie, J. Huang, and Z. Liang, "Vibration reduction for flexible systems by command smoothing," Mechanical Systems and Signal Processing, vol. 39, no. 1-2, pp. 461-470, 2013.

[3] K.-B. Lee and F. Blaabjerg, "An improvement of speed control performances of a two-mass system using a universal approximator," Electrical Engineering, vol. 89, no. 5, pp. 389-396, 2007.

[4] K. Szabat and T. Orlowska-Kowalska, "Vibration suppression in a two-mass drive system using PI speed controller and additional feedbacks - Comparative study," IEEE Transactions on Industrial Electronics, vol. 54, no. 2, pp. 1193-1206, 2007.

[5] D. Xie, D. Qu, and F. Xu, "Design of Ho feedback controller and IP-position controller of PMSM servo system," in Proceedings of the IEEE International Conference on Mechatronics and Automation, ICMA 2005, pp. 578-583, can, August 2005.

[6] M. Kaminski, "Neural network speed controller for drive system with elastic joint," in Proceedings of the IEEE EuroCon 2013, pp. 2080-2085, Croatia, July 2013.

[7] Y. Qiao, L. Zhou, and C. Ma, "Polynomial-based inertia ratio controller design for vibration suppression in two-mass system," in Proceedings of the 39th Annual Conference of the IEEE Industrial Electronics Society, IECON 2013, pp. 3687-3692, Austria, November 2013.

[8] S. Villwock and M. Pacas, "Application of the welch-method for the identification of two- and three-mass-systems," IEEE Transactions on Industrial Electronics, vol. 55, no. 1, pp. 457-466, 2008.

[9] H. Zoubek and M. Pacas, "An identification method for multimass-systems in speed-sensorless operation," in Proceedings of the 2011 IEEE International Symposium on Industrial Electronics, ISIE 2011, pp. 1895-1900, Poland, June 2011.

[10] M. Ruderman, W. Maebashi, and M. Iwasaki, "Semi-dual loop control of two-mass actuator system using Luenberger state observer," in Proceedings of the 39th Annual Conference of the IEEE Industrial Electronics Society, IECON 2013, pp. 6563-6568, Austria, November 2013.

[11] C. Ma, J. Cao, and Y. Qiao, "Polynomial-method-based design of low-order controllers for two-mass systems," IEEE Transactions on Industrial Electronics, vol. 60, no. 3, pp. 969-978, 2013.

[12] J. Ye, J. Cui, and Z. Chu, "Experimental study of vibration suppression for uncertain two-mass torsional system," in Proceedings of the 12th IEEE International Conference on Control and Automation, ICCA 2016, pp. 996-1001, npl, June 2016.

[13] B. Liang, S. Iwnicki, A. Ball, and A. E. Young, "Adaptive noise cancelling and time-frequency techniques for rail surface defect detection," Mechanical Systems and Signal Processing, vol. 54-55, pp. 41-51, 2015.

[14] Z. K. Peng, W. M. Zhang, Z. Q. Lang, G. Meng, and F. L. $\mathrm{Chu}$, "Time-frequency data fusion technique with application to vibration signal analysis," Mechanical Systems and Signal Processing, vol. 29, pp. 164-173, 2012.

[15] F. Al-Badour, M. Sunar, and L. Cheded, "Vibration analysis of rotating machinery using time-frequency analysis and wavelet techniques," Mechanical Systems and Signal Processing, vol. 25, no. 6, pp. 2083-2101, 2011. 


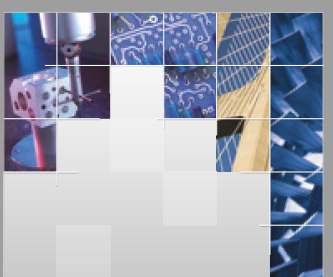

\section{Enfincering}
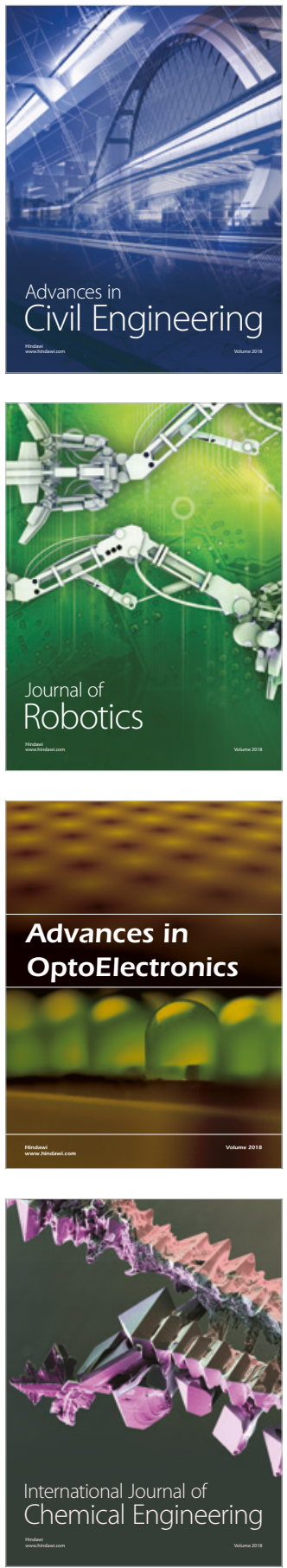

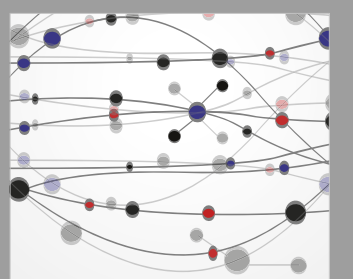

\section{Rotating \\ Machinery}

The Scientific World Journal

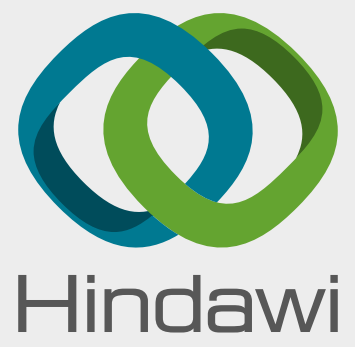

Submit your manuscripts at

www.hindawi.com
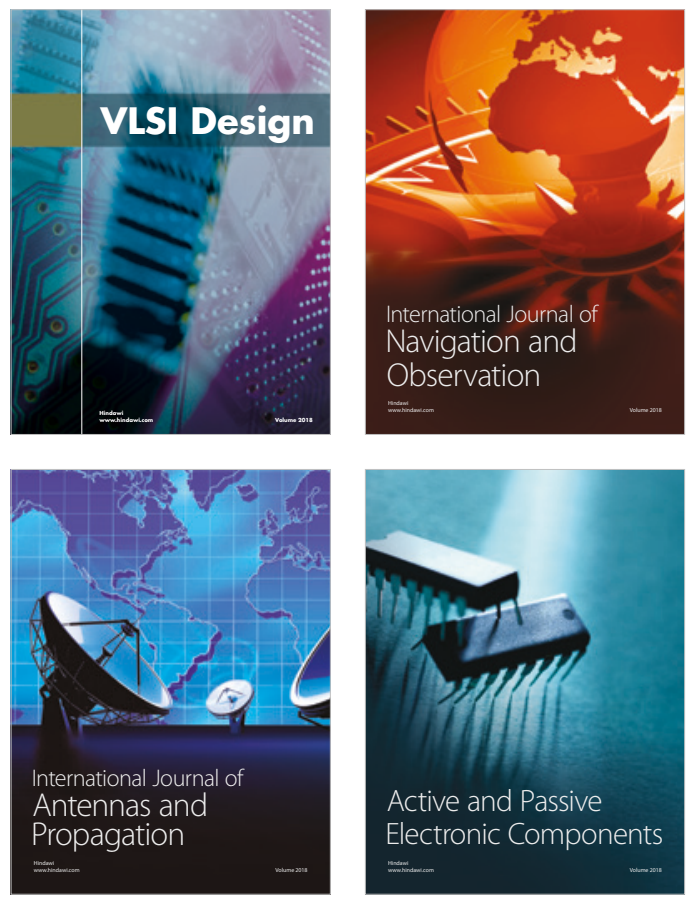
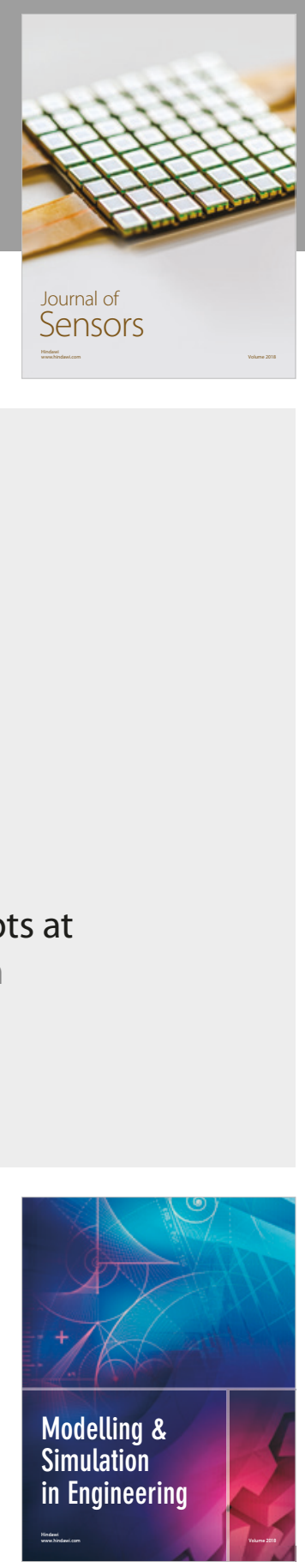

\section{Advances \\ Multimedia}
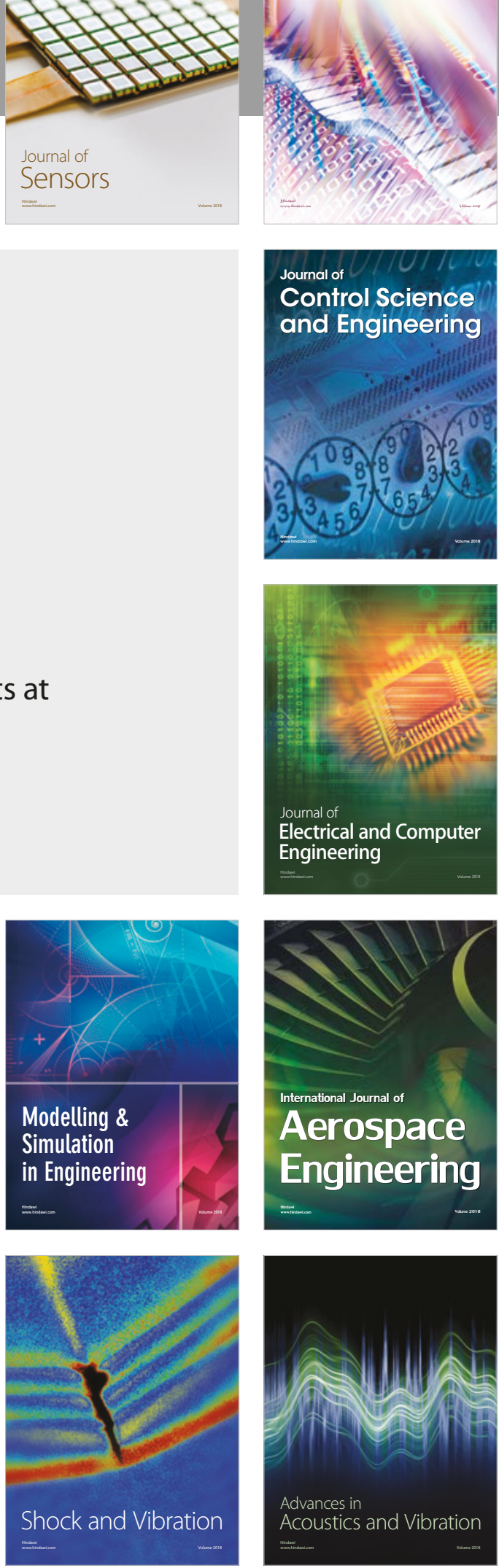